\title{
Therapeutic Concepts for Oligometastatic Gastrointestinal Tumours
}

\author{
Jens Ricke $^{\mathrm{a}}$ Christoph Benedikt Westphalen ${ }^{\mathrm{b}} \quad$ Max Seidensticker ${ }^{\mathrm{a}}$ \\ ${ }^{a}$ Klinik und Poliklinik für Radiologie, LMU Klinikum, Munich, Germany; ${ }^{b}$ Medizinische Klinik und Poliklinik III und CCC \\ München ${ }^{\text {LMU }}$, Klinikum der Universität München, LMU München, Munich, Germany
}

\section{Keywords}

Oligometastases · Gastrointestinal tumours · Ablation · Locoregional treatment $\cdot$ Image-guided intervention

\begin{abstract}
Background: Clinical trials have proven a survival benefit from applying local therapies for oligometastatic cancers of various origin. Summary: Today, the definition of oligometastatic disease is based on limited lesion numbers and organ systems involved. Treatment guidelines by the European Organisation for Research and Treatment of Cancer (EORTC), European Society for Medical Oncology (ESMO) and several other groups suggest a threshold of up to 5 tumours. Established biological markers indicating the aggressiveness of a given tumour (and therefore suggesting local treatment only or the addition of or complete switch to systemic therapies) are missing, except for disease-free survival, the only recommended parameter for patient selection beyond lesion count. Key Message: The following article discusses clinical implications as well as local techniques established for the treatment of oligometastatic disease.
\end{abstract}

(c) 2020 S. Karger AG, Basel

\section{Introduction}

The term "oligometastasis" has first been introduced by Hellman and Weichselbaum [1]. Primarily, it had been intended as defining a curative stage of metastatic disease eligible for local treatment. Thresholds separating a curative from a palliative disease character remain undefined. At present, oligometastatic disease is defined by a limited number of tumours and affected organ systems [2]. However, variables measuring the biological aggressiveness of tumours are missing $[3,4]$.

Simply limiting the assessment to tumour number and spread carries the inherent risk of allocating patients to local treatments who will relapse within a short time and might have benefitted more from systemic treatments. Still, the number of metastases as the single determinant for the use of local ablative techniques has proven to be a valid predictive factor even when targeting heterogeneous tumour entities, including rather aggressive entities such as breast, prostate and non-small cell lung cancer. Palma and colleagues $[5,6]$ included 99 patients with up to 5 metastases, with some of the patients in the cohort having a controlled primary tumour in situ. The patients were randomised to local ablation by stereotactic body radiation therapy, whilst the control group received standard therapy. Both overall survival and quality of life were significantly improved in the local treatment arm (overall survival: HR 0.57 [95\% CI: 0.30-1.10]). These results sug- 
gest that biological determinants - even if assessed by tumour number only - may well exceed the value of established histopathological classifications for determining a successful clinical therapeutic strategy.

\section{Patient Selection for Local Treatment Strategies in Oligometastatic Disease}

\section{Biological Behaviour}

Curative treatment strategies for metastatic disease were first introduced via the evolution of liver resection in colorectal cancer. Measured in current terms, the benchmark proposed for liver surgery by the Fong score may be considered defensive and has lost clinical relevance over the years $[7,8]$. The Fong score combines the number of metastases, the disease-free interval, lymphatic involvement of the primary, the size of the liver metastases and carcinoembryonic antigen for risk stratification by estimating a patient's likely survival. However, lung and lymph node metastases, and even limited peritoneal seeding, were considered eligible for local treatment in the case of colorectal cancer, often in combination with neoadjuvant or other systemic treatments $[3,9]$.

Just recently, a dedicated European Organisation for Research and Treatment of Cancer (EORTC) board led by Guckenberger has established a status update on oligometastatic disease and its clinical implications [4]. The authors introduced the concept of genuine versus acquired oligometastatic disease, i.e. a limited number of metastases technically eligible for local therapy at diagnosis versus a disease burden after response to systemic therapy. Different models, specifically after systemic therapy, were discussed, such as disease staging following a mixed response within the numeric limits of oligometastatic disease. According to the EORTC group, the tumour biology in genuine oligometastatic disease may be more benign than that after a successful response of oligometastatic disease to induction chemotherapy [4]. One reason for a negative bias towards higher biological aggressiveness could be the selection of chemorefractory clones, a very high tumour load or refractoriness to pharmacological approaches induced by the harbouring organ [1].

In contrast, based on preliminary phase II data, clinical decision-making tends to concentrate local therapies such as resection or ablation on patients displaying favourable responses after chemotherapy. This concept is primarily applied when histology implies that a primary tumour may generally be rather unfavourable for local treatment $[10,11]$. However, such reasoning may run the risk of mixing prognosis and prediction. If understood as a consolidation strategy rather than a curative option for oligometastasis, local treatments have demonstrated a high clinical benefit, allowing patients to experience prolonged periods of rest from systemic treatment [12].

The sole undisputed determinant of benign biological behaviour today is disease-free survival. Synchronous metastases are considered unfavourable $[5,13]$.

\section{Pattern of Distribution}

In a variety of tumours, the distribution pattern of metastatic disease represents an indication of their malignant potential. Patients with isolated (even multiple) colorectal liver metastases may reach long-term survival beyond 10 years if these metastases are ablated or resected [14]. Even limited numbers of lymph node metastases beyond local spread to the hepatoduodenal ligament may preclude 5 -year survival [15]. However, in such patients, resection or ablation frequently are considered debulking rather than a curative approach to oligometastatic disease [16].

Another example of a favourable metastatic pattern in gastrointestinal tumours is pancreatic adenocarcinoma with isolated pulmonary metastases. Despite the fact that adenocarcinoma of the pancreas usually demonstrates aggressive systemic spread once progressed beyond local stages, patients developing metastatic spread exclusively to the lungs frequently attain long-term survival after pulmonary resection or ablation [17] and represent a favourable subgroup [18].

\section{Biomarkers and Genetic Signatures}

Pitroda et al. [19] reported on a genomic signature determining the biological aggressiveness of colorectal cancer. Patients with numerically oligometastatic colorectal cancer underwent molecular subtyping, leading to a recurrence rate as low as $20 \%$ after 10 years if displaying a favourable signature. Stratification into high-, intermediate- and low-risk groups yielded a 10-year survival of 19, 45 and $94 \%$.

Previous studies have identified BRAF mutation as an indicator of higher biological aggressiveness in colorectal cancer, which in theory disqualifies patients from local treatment. However, colorectal cancer metastases carrying BRAF mutations frequently demonstrate a low sensitivity to systemic chemotherapy. In such inherently chemorefractory patients, limited disease spread such as in oligometastatic patterns may allow for the palliative use of local therapies when other options are lacking [20,21]. MSI-high (high-microsatellite instability) colorectal cancer displays lymph node or other distant metastases significantly less frequently [22]. However, the metastatic pattern of MSI-high as well as of $B R A F$-mutated colorectal cancers frequently includes peritoneal spread or lymph node metastases. Isolated liver metastases appear with low frequency [23]. Mutations of the KRAS gene are associated with higher numbers of lung and brain metastases, and liver-only disease is rare $[20,24]$. 


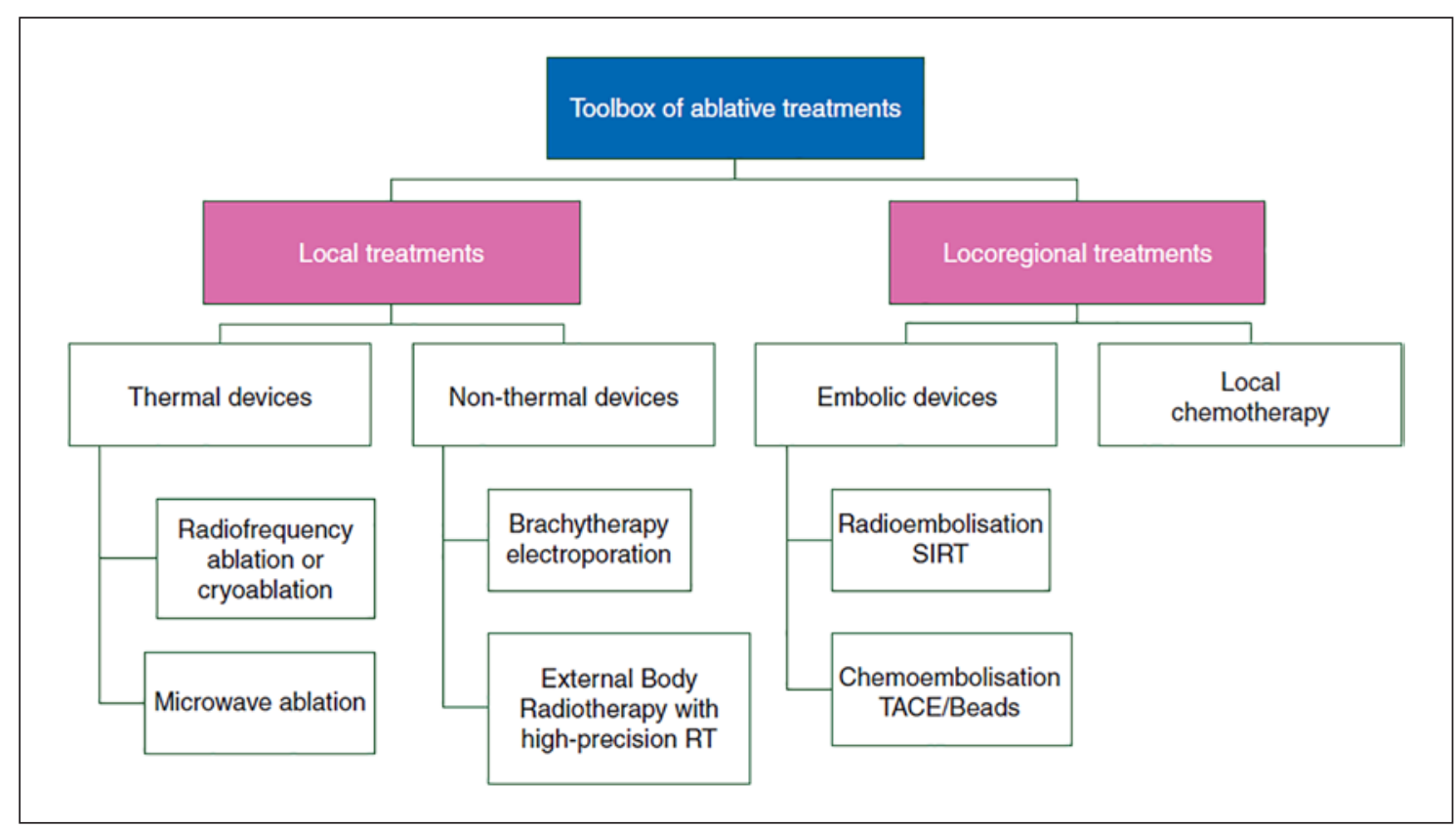

Fig. 1. The toolbox of local image-guided treatments as published by the ESMO for oligometastatic colorectal cancer (from Van Cutsem et al. [3]; originally designed by M. Pech, 4th Annual Congress of the Sachsen-Anhaltinische Krebsgesellschaft, Magdeburg, May 2011). This concept does not include minimally invasive surgical techniques such as laparoscopic or robot-assisted resection. RT, radiation therapy; SIRT, selective internal radiotherapy $\left({ }^{90} \mathrm{Y}\right.$ radioembolisation); TACE, transarterial chemoembolisation.

Discrimination between oligo- and polymetastatic disease by mRNA has been demonstrated in a predictive study of resected lung metastases originating from a variety of primary tumours. The study included 63 patients with up to 5 lung metastases undergoing lung resection with curative intent. Patients developing more than 5 new lung metastases within 4 months were considered polymetastatic. Discrimination of the polymetastatic patient group by mRNA expression was significant [25].

\section{The Toolbox of Local Treatments}

In 2016, the European Society for Medical Oncology (ESMO) published guidelines for the treatment of metastatic colorectal cancer including recommendations for the use of local treatments. Interestingly, the group reached a consensus on a "toolbox concept" of local ablative, image-guided treatments. The recommendation was based on patient characteristics, technical factors and local expertise; an appropriate technique for ablation should be chosen by interdisciplinary local consensus such as a tumour board [3]. Figure 1 illustrates the various techniques stratified by treatment strategy. Local ablation, including thermal or high conformal irradiation, is used with the aim of full remission, i.e. total tumour destruction. With a more diffuse tumour spread limited to a sin- gle organ (usually the liver), local ablation may not be feasible. In such cases, locoregional therapies such as selective internal radiotherapy $\left({ }^{90} \mathrm{Y}\right.$ radioembolisation) or transarterial chemoembolisation aim at partial remission, with cases of full remission being rather anecdotal (Fig. 1).

The most obvious advantage of local or locoregional treatments is good tolerability even by patients with comorbidities, as well as a relatively low complication rate. Seidensticker et al. [26] published a study on 266 patients undergoing local ablation or locoregional treatments of metastatic colorectal cancer either alone, in combination or sequentially. This cohort comprised patients with heterogeneous disease stages ranging from single liver lesions to multiple organ system involvement, commonly following multiple chemotherapy lines. Comorbidity was measured using the Charlson comorbidity index (CCI) and the Charlson age-comorbidity index (CACI), excluding metastatic colorectal cancer as the index disease. Age $\geq 70$ years did not influence survival after local therapies. Similarly, the CCI or CACI did not affect patients' prognoses in multivariate analyses [26]. Mohnike et al. [27] assessed a total of 781 tumour ablations (radiofrequency ablation, $n=112$; interstitial brachytherapy, $n=669$ ) in 446 patients for complications. The all-cause 30 -day mortality rate was $0.5 \%$ and the all-cause 90 -day mortality rate was $2.3 \%$ in a study population also including $12.5 \%$ 
of cirrhotic patients. This compares favourably with a study by Filmann et al. [28], reporting a hospital mortality rate of $5.8 \%$ among 110,332 patients undergoing liver procedures carried out between 2010 and 2015. The mortality rate among 17,574 patients undergoing major hepatic procedures was $10.4 \%$. Patients who had surgery for colorectal liver metastases had the lowest mortality rate among those with malignancy (5.5\%), followed by patients with gallbladder cancer (7.1\%), those with hepatocellular carcinoma $(9.3 \%)$ and those with intrahepatic cholangiocarcinoma (11\%). The mortality rate after resection of colorectal liver metastases was $4.6 \%$ in veryhigh-volume centres compared with $7.5 \%$ in very-lowvolume hospitals $(p<0.001)$.

\section{Therapeutic Strategies}

Data on tolerability, complications and mortality after surgery or local ablation suggest that surgical resection is strongly recommended if the treatment intent is clearly curative. However, oligometastatic disease defined by lesion number only may well include patients at risk of early disease relapse, and biological markers determining the likelihood of such early progression are missing. The lack of a threshold between palliation and debulking has strengthened the role of image-guided techniques $[5,26]$. The CLOCC study may serve as an example of an oligometastatic patient cohort on an unclear threshold towards palliative intent [29]. CLOCC recruited patients with up to 10 liver metastases from chemotherapy-naive colorectal cancer. Initially rated irresectable by a hepatobiliary surgeon, all patients were scheduled to receive systemic chemotherapy. The experimental arm was randomised to local ablation (additional simultaneous liver resection was performed on $50 \%$ of the subjects) before initiation of the systemic therapy. The ablative strategy aimed for complete ablation/resection. The survival benefit for the patients in the combination arm was significant (HR 0.58 [95\% CI 0.38-0.88], $p=0.01$ ). Interestingly, the survival curves from Kaplan-Maier analysis began to separate at 4 years, with fewer than $50 \%$ of the patients left at risk in the combination arm, indicating a high proportion of rather palliatively treated patients among the overall cohort. The 8 -year survival rate was $35.9 \%$ (vs. 8.9\%) [29].

It remains controversial whether local treatments for oligometastatic colorectal cancer may yield a benefit by debulking or "deepness of response" [16], and there certainly is no evidence for other gastrointestinal cancers. Of interest are considerations about clonal selection pressure, specifically if the oligometastatic state has been reached by previous systemic chemotherapy or mixed responses [4]. In such cases originating from unfavourable clonal selection, ablation or resection, overcoming chemotherapy refractoriness in non- or mixed responders, may yield a significant benefit [30].

\section{Conclusions}

Clinical trials have proven survival benefits from applying local therapies for oligometastatic cancers of various origin. At present, the definition of oligometastatic disease is based on limited lesion numbers and organ systems involved. The EORTC as well as several other groups issuing treatment guidelines on a variety of tumours suggest a threshold of up to 5 tumours. Established biological markers indicating the aggressiveness of a given tumour (and therefore suggesting local treatment only or the addition of or complete switch to systemic therapies) are missing, except for disease-free survival, the only recommended parameter for patient selection beyond lesion count. In the era of precision oncology, interdisciplinary approaches are needed to fill this evidence gap in order to better tailor multimodal treatment to our patients.

\section{Conflict of Interest Statement}

The authors declare no conflict of interest.

\section{Funding Sources}

No funding was received.

\section{Author Contributions}

Literature search and drafting of the manuscript: J.R.; literature search and manuscript editing: C.B.W. and M.S.

\section{References}

1 Hellman S, Weichselbaum RR. Importance of local control in an era of systemic therapy. Nat Clin Pract Oncol. 2005 Feb;2(2):60-1.

2 Weichselbaum RR, Hellman S. Oligometastases revisited. Nat Rev Clin Oncol. 2011 Jun; 8(6):378-82.

3 Van Cutsem E, Cervantes A, Adam R, Sobrero A, Van Krieken JH, Aderka D, et al. ESMO consensus guidelines for the management of patients with metastatic colorectal cancer. Ann Oncol. 2016 Aug;27(8):1386-422.

4 Guckenberger M, Lievens Y, Bouma AB, Collette L, Dekker A, deSouza NM, et al. Characterisation and classification of oligometastatic disease: a European Society for Radiotherapy and Oncology and European Organisation for Research and Treatment of Cancer consensus recommendation. Lancet Oncol. 2020 Jan;21(1):e18-28. 
5 Palma DA, Olson R, Harrow S, Gaede S, Louie AV, Haasbeek C, et al. Stereotactic ablative radiotherapy versus standard of care palliative treatment in patients with oligometastatic cancers (SABR-COMET): a randomised, phase 2, open-label trial. Lancet. 2019 May; 393(10185):2051-8.

6 Olson R, Senan S, Harrow S, Gaede S, Louie A, Haasbeek C, et al. Quality of Life Outcomes after Stereotactic Ablative Radiation Therapy (SABR) versus Standard of Care Treatments in the Oligometastatic Setting: A Secondary Analysis of the SABR-COMET Randomized Trial. Int J Radiat Oncol Biol Phys. 2019 Dec; 105(5):943-7.

7 Fong Y, Fortner J, Sun RL, Brennan MF, Blumgart LH. Clinical score for predicting recurrence after hepatic resection for metastatic colorectal cancer: analysis of 1,001 consecutive cases. Ann Surg. 1999 Sep;230(3):309-18.

8 Iida T, Nomori H, Shiba M, Nakajima J, Okumura S, Horio $\mathrm{H}$, et al.; Metastatic Lung Tumor Study Group of Japan. Prognostic factors after pulmonary metastasectomy for colorectal cancer and rationale for determining surgical indications: a retrospective analysis. Ann Surg. 2013 Jun;257(6):1059-64.

9 Saxena A, Valle SJ, Liauw W, Morris DL. Limited synchronous hepatic resection does not compromise peri-operative outcomes or survival after cytoreductive surgery and hyperthermic intraperitoneal chemotherapy. J Surg Oncol. 2017 Mar;115(4):417-24.

10 Gomez DR, Blumenschein GR Jr, Lee JJ, Hernandez $\mathrm{M}$, Ye $\mathrm{R}$, Camidge $\mathrm{DR}$, et al. Local consolidative therapy versus maintenance therapy or observation for patients with oligometastatic non-small-cell lung cancer without progression after first-line systemic therapy: a multicentre, randomised, controlled, phase 2 study. Lancet Oncol. 2016 Dec;17(12):1672-82.

11 Crippa S, Bittoni A, Sebastiani E, Partelli S, Zanon S, Lanese A, et al. Is there a role for surgical resection in patients with pancreatic cancer with liver metastases responding to chemotherapy? Eur J Surg Oncol. 2016 Oct; 42(10):1533-9.

12 Benoist S, Brouquet A, Penna C, Julié C, El Hajjam M, Chagnon S, et al. Complete response of colorectal liver metastases after chemotherapy: does it mean cure? J Clin Oncol. 2006 Aug;24(24):3939-45.
13 Dünschede F, Will L, von Langsdorf C, Möhler M, Galle PR, Otto G, et al. Treatment of metachronous and simultaneous liver metastases of pancreatic cancer. Eur Surg Res. 2010;44(3-4):209-13.

14 Van Tilborg AA, Meijerink MR, Sietses C, Van Waesberghe JH, Mackintosh MO, Meijer $\mathrm{S}$, et al. Long-term results of radiofrequency ablation for unresectable colorectal liver metastases: a potentially curative intervention. Br J Radiol. 2011 Jun;84(1002):556-65.

15 Adam R, de Haas RJ, Wicherts DA, Aloia TA, Delvart V, Azoulay D, et al. Is hepatic resection justified after chemotherapy in patients with colorectal liver metastases and lymph node involvement? J Clin Oncol. 2008 Aug; 26(22):3672-80

16 Mannsmann U, Sartorius U, Laubenender R. Quantitative analysis of the impact of deepness of response on post-progression survival time following first line treatment in patients with mCRC. J Clin Oncol. 2013;31(15_suppl): 3630 .

17 Arnaoutakis GJ, Rangachari D, Laheru DA, Iacobuzio-Donahue CA, Hruban RH, Herman JM, et al. Pulmonary resection for isolated pancreatic adenocarcinoma metastasis: an analysis of outcomes and survival. J Gastrointest Surg. 2011 Sep;15(9):1611-7.

18 Kruger S, Haas M, Burger PJ, Ormanns S, Modest DP, Westphalen CB, et al. Isolated pulmonary metastases define a favorable subgroup in metastatic pancreatic cancer. Pancreatology. 2016 Jul-Aug;16(4):593-8.

19 Pitroda SP, Khodarev NN, Huang L, Uppal A, Wightman SC, Ganai S, et al. Integrated molecular subtyping defines a curable oligometastatic state in colorectal liver metastasis. Nat Commun. 2018 May;9(1):1793.

20 Lipsyc M, Yaeger R. Impact of somatic mutations on patterns of metastasis in colorectal cancer. J Gastrointest Oncol. 2015 Dec;6(6): 645-9.

21 Roth AD, Tejpar S, Delorenzi M, Yan P, Fiocca R, Klingbiel D, et al. Prognostic role of KRAS and BRAF in stage II and III resected colon cancer: results of the translational study on the PETACC-3, EORTC 40993, SAKK 6000 trial. J Clin Oncol. 2010 Jan;28(3):466-74.

22 Malesci A, Laghi L, Bianchi P, Delconte G, Randolph A, Torri V, et al. Reduced likelihood of metastases in patients with microsatellite-unstable colorectal cancer. Clin Cancer Res. 2007 Jul;13(13):3831-9.
23 Tran B, Kopetz S, Tie J, Gibbs P, Jiang ZQ, Lieu $\mathrm{CH}$, et al. Impact of BRAF mutation and microsatellite instability on the pattern of metastatic spread and prognosis in metastatic colorectal cancer. Cancer. 2011 Oct;117(20): 4623-32.

24 Kim MJ, Lee HS, Kim JH, Kim YJ, Kwon JH, Lee JO, et al. Different metastatic pattern according to the KRAS mutational status and site-specific discordance of KRAS status in patients with colorectal cancer. BMC Cancer. 2012 Aug; 12:347.

25 Lussier YA, Khodarev NN, Regan K, Corbin $\mathrm{K}, \mathrm{Li} \mathrm{H}$, Ganai S, et al. Oligo- and polymetastatic progression in lung metastasis(es) patients is associated with specific microRNAs. PLoS One. 2012;7(12):e50141.

26 Seidensticker R, Damm R, Enge J, Seidensticker M, Mohnike K, Pech M, et al. Local ablation or radioembolization of colorectal cancer metastases: comorbidities or older age do not affect overall survival. BMC Cancer. 2018 Sep;18(1):882.

27 Mohnike K, Sauerland H, Seidensticker M, Hass P, Kropf S, Seidensticker R, et al. Haemorrhagic Complications and Symptomatic Venous Thromboembolism in Interventional Tumour Ablations: The Impact of Peri-Interventional Thrombosis Prophylaxis. Cardiovasc Intervent Radiol. 2016 Dec;39(12):171621.

28 Filmann N, Walter D, Schadde E, Bruns C, Keck T, Lang H, et al. Mortality after liver surgery in Germany. Br J Surg. 2019 Oct;106(11): 1523-9.

29 Ruers T, Van Coevorden F, Punt CJ, Pierie JE, Borel-Rinkes I, Ledermann JA, et al.; European Organisation for Research and Treatment of Cancer (EORTC); Gastro-Intestinal Tract Cancer Group; Arbeitsgruppe Lebermetastasen und Tumoren in der Chirurgischen Arbeitsgemeinschaft Onkologie (ALMCAO); National Cancer Research Institute Colorectal Clinical Study Group (NCRI CCSG). Local Treatment of Unresectable Colorectal Liver Metastases: Results of a Randomized Phase II Trial. J Natl Cancer Inst. 2017 Sep;109(9):djx015.

30 Nikbakht H, Jessa S, Sukhai MA, Arseneault M, Zhang T, Letourneau L, et al. Latency and interval therapy affect the evolution in metastatic colorectal cancer. Sci Rep. 2020 Jan; 10(1):581. 